\title{
Grabaciones de situaciones de aula para la formación del profesorado
}

\author{
María José Gil Quílez ${ }^{1}$, Ma Begoña Martínez Peña ${ }^{1}$ y Silvina Cordero ${ }^{2}$ \\ ${ }^{1}$ Grupo Beagle (IUCA) Departamento de Didáctica de las Ciencias Experimentales, \\ Facultad de Educación, Universidad de Zaragoza, España \\ ${ }^{2}$ Facultad de Humanidades y Ciencias de la Educación y Grupo de Didáctica de las \\ Ciencias. Universidad Nacional de La Plata, Argentina
}

[Recibido el 10 de marzo de 2017, aceptado el 19 de mayo de 2017]

En este trabajo se presentan los resultados de la valoración crítica realizada por estudiantes de magisterio de Educación Primaria ante dos grabaciones de aula realizadas por maestros en formación durante sus prácticas escolares. Los futuros maestros, tras el visionado de las mismas, realizan un análisis de dicha actuación desde el marco de la indagación. Perciben estas actuaciones de aula como algo próximo y factible, y encuentran interesantes este tipo de actividades por lo que se implican en su análisis.

Palabras-clave: Análisis de vídeo; maestros; indagación; formación de profesorado.

\section{Using video recordings to teach teachers}

In this work we present the results of the critical evaluation carried out by preservices teachers of Primary Education of two classroom recordings made by teachers in formation during their school practices. The future teachers, after the viewing of the same ones, make an analysis of this action from the framework of the inquiry. They perceive their classroom as something near and feasible, and find this type of activity useful, so they get involved in their analysis.

Keywords: Video analysis; Teachers; inquiry; teacher development.

Para citar el artículo. Gil, M.J., Martínez, M.B. y Cordero, S. (2017). Grabaciones de situaciones de aula para la formación del profesorado. Ápice. Revista de Educación Científica, 1(1), 58-73. DOI: https://doi.org/10.17979/arec.2017.1.1.2005

Contacto.quilez@unizar.es, bpena@unizar.es, silvina.cordero.protto@gmail.com 


\section{Introducción}

La Didáctica de las Ciencias se ocupa de estudiar los elementos que intervienen en la enseñanza aprendizaje de las ciencias (alumnado, profesorado, saber, medio) y sus relaciones (planteamiento de preguntas, problemas, actividades, etc.), con el objetivo de comprender cómo se producen los aprendizajes para conseguir que estos sean efectivos. Pero en la formación de los futuros maestros sobre enseñanza de las ciencias, tras años de experiencia, seguimos constatando que lo último que les preocupa es si finalmente aprenden ciencias sus alumnos. Opinan que los contenidos son fáciles (Cortés et al., 2012) y que la motivación del alumnado, el trabajo en equipo y las metodologías activas (independientemente del significado personal que cada uno le atribuye) es definitivo para enseñar ciencias. Por supuesto, todo ello es necesario, pero no tienen en cuenta la materia sobre la que están trabajando a la hora de planificar cómo motivar, organizar el trabajo en equipo o la metodología a emplear. Sería deseable que dicha planificación se desarrollase dentro de una propuesta de indagación dirigida, es decir, que ante fenómenos cotidianos, los futuros maestros planteen preguntas que motiven el desarrollo de una pequeña investigación que implique un trabajo en equipo entre el alumnado para buscar soluciones a dichas preguntas (Cañal, 2007).

La enseñanza por indagación constituye un desafío para los docentes noveles y en formación, no sólo por los conocimientos y procedimientos científicos que involucra (Gil Quílez, Martínez Peña, De la Gándara, Calvo y Cortés, 2008), sino también por las variantes respecto de la gestión del aula desde el punto de vista organizativo que implica. Enfrentados a sus primeras prácticas, los principiantes apelan frecuentemente a metodologías menos participativas, como la clase magistral o el trabajo individual con fichas de lectura a fin de asegurarse el "control" de la clase. También al analizar y criticar clases de ciencias, los maestros en formación centran su atención en "el barullo", "el descontrol" y la "pérdida de atención" que las actividades manipulativas, experiencias o experimentos generan en el alumnado.

Estos posicionamientos surgirían, por un lado, de una aproximación limitada al enfoque de enseñanza por indagación, que no les permite imaginar matices, variantes y niveles en la utilización de esta metodología. Por otro lado, de sus dificultades para articular teoría y práctica, vinculando aprendizajes de asignaturas de la formación pedagógica general, como la programación didáctica por ejemplo, con el tratamiento de un contenido específico y un contexto de aula real, con todas sus complejidades.

En un intento por atender a estas necesidades formativas, hemos implementado un proyecto en el que, por una parte, se analizaron los vídeos de las actuaciones de dos estudiantes durante las prácticas escolares en un centro docente $y$, por otra, se utilizaron dichos vídeos como instrumento para trabajar en el aula con el alumnado de Magisterio la formación inicial en Didáctica de las Ciencias.

Los resultados obtenidos en investigaciones que proponen el análisis y la valoración crítica de propuestas y materiales señalan que se trata de una actividad positiva y recomendada en la formación de maestros (Abell, Anderson y Bryan, 1998; Davis, 2006; Duncan, Pilitsis y Piegaro, 2010). Al realizar la valoración se ponen de manifiesto los criterios empleados y la idea que subyace sobre la enseñanza aprendizaje de las ciencias. El paso siguiente sería analizar una clase en contexto, es decir, no solo la propuesta y los materiales sino lo que finalmente ocurre en el aula. 
Cuando trabajamos con los futuros maestros de educación primaria pretendemos, entre otras cuestiones, que sean capaces de proponer actividades y secuencias de actividades que luego llevarán al aula de primaria. En sus propuestas didácticas siguen las orientaciones recibidas, pero acaban siendo lineales y no queda reflejado en ellas cómo planifican la interacción con los alumnos que obliga a tomar decisiones a lo largo de la clase, de manera que finalmente resulta difícil que propongan clases en las que se produzca dicha interacción. Consideramos que analizar el valor educativo de materiales docentes y propuestas didácticas es un aspecto importante de su formación, ya que les capacita para revisar y adaptar dichos materiales y propuestas con el objetivo de promover en sus alumnos el aprendizaje de las ciencias. Este hecho es especialmente relevante en maestros en formación ya que, ante su falta de experiencia, resultan más dependientes de materiales ya elaborados.

En este trabajo presentamos los resultados de la valoración crítica realizada por estudiantes de magisterio de Educación Primaria ante dos situaciones de aula. Elegimos estos vídeos porque había mucha interacción, protagonismo del alumnado, y eran clases pensadas, planificadas, con numerosas experiencias y materiales. Eran docentes que ya habían cursado toda su formación teórica y habían trabajado la enseñanza por indagación en las asignaturas de DCE. Los futuros maestros, tras el visionado de las actuaciones de aula de sus compañeros, durante las prácticas escolares, realizan una crítica de dicha actuación desde el marco de la indagación. Queremos conocer, frente una situación real de aplicación de una propuesta de indagación, qué criterios tienen en cuenta para valorar si se ha hecho o no indagación, y qué es lo que consideran importante de la clase.

\section{Marco teórico}

En la actualidad existe un consenso sobre la necesidad de modificar la imagen de la ciencia trasmitida por la enseñanza tradicional, que como señalan algunos autores (Vázquez Alonso, Acevedo Díaz y Manassero Mas, 2004), se ha considerado como un conjunto de conocimientos acabados, definitivos y dogmáticos, que no coincide con la realidad de la naturaleza de la ciencia (Acevedo Díaz, 2008). De manera general se puede decir que la naturaleza de la ciencia aborda cuestiones sobre qué es la ciencia, cómo trabajan los científicos y qué relaciones se establecen entre la ciencia y la sociedad (Guisasola y Morentín, 2007). La participación en prácticas científicas, como la planificación y puesta en práctica de investigaciones, constituye un aspecto clave del aprendizaje de las ciencias y debería permitir un acercamiento al conocimiento de la naturaleza de la ciencia. Cuando los estudiantes se implican en prácticas científicas, la realización de experimentos, toma de datos, registro de pruebas, discusión de resultados y diseño de nuevos experimentos se convierten en la base del aprendizaje científico. Según Crujeiras y Jiménez-Aleixandre (2015), las prácticas científicas se pueden agrupar en tres tipos: la indagación, la evaluación del conocimiento (es decir, la argumentación) y la construcción de explicaciones y modelos.

La enseñanza por indagación constituye un enfoque ampliamente valorado y recomendado tanto desde la investigación en didáctica de las ciencias como desde las últimas reformas curriculares (NATIONAL RESEARCH COUNCIL, 2000; MEC, 2007; Rocard et al., 2007). Este enfoque propone la implementación de actividades que permitan al alumnado experimentar, con las adaptaciones necesarias al contexto escolar, procesos equiparables a los de la investigación científica. Pero no existe una única forma de 
desarrollar la enseñanza por indagación. Windschitl (2003) establece, para el contexto escolar, cuatro niveles de indagación:

- Experiencias de confirmación, que sería el nivel más bajo, en el que los estudiantes, siguiendo el procedimiento diseñado por el profesor, verifican principios científicos conocidos.

- Indagación estructurada, en la que el profesorado presenta una pregunta y proporciona un procedimiento a seguir con el fin de completar la investigación.

- Indagación guiada, en la que se proporciona a los estudiantes un problema para investigar, pero deben ser ellos los que decidan los métodos para resolver el problema.

- Indagación abierta, donde los estudiantes plantean sus propias preguntas y diseñan sus propias investigaciones.

Para decidir el nivel con el que trabajar en el aula hay que considerar el grado de desarrollo de habilidades y competencias del alumnado. De esta manera, en los estadios iniciales resultan más eficaces los enfoques estructurados para que los alumnos construyan modelos, que los guiados y que los abiertos (Kirschner, Sweller y Clark, 2006). Estos últimos niveles habría que aplicarlos cuando el alumnado goza ya de cierto grado de conocimiento (saber y saber hacer).

Estudios realizados con profesorado de educación secundaria, tanto en activo como en formación, ponen en evidencia que relacionan la enseñanza por indagación con "actividades divertidas" para el alumnado, lo que llevaría a pensar que la ven más como un recurso para motivar que para potenciar o mejorar el aprendizaje (Abril et al., 2014). Por otra parte, trabajos realizados con maestros en formación (Cortés Gracia y De la Gándara Gómez, 2006; Gil Quílez, et al., 2008; Lucero, Valcke y Schellens, 2013; Crujeiras y Puig, 2014; Vílchez González y Bravo Torija, 2015) señalan que los futuros docentes, cuando tienen que desarrollar actividades de indagación, encuentran dificultades para elegir la pregunta a investigar, planificar el diseño experimental e identificar las variables que intervienen. Algunos autores relacionan los problemas para utilizar la enseñanza por indagación con una falta de conocimiento acerca de qué es la indagación y cómo ha de implementarse en el aula (Cuevas, Lee, Hart y Deakort, 2005; McDonald y Butler Soger, 2008). Como sugieren Vílchez González y Bravo Torija (2015), después de analizar las limitaciones en los conocimientos de los docentes en formación respecto de las etapas de una indagación científica, resulta fundamental, en la formación universitaria del profesorado de ciencias naturales, proponer y analizar ejemplos de actividades considerando las características de la indagación escolar, así como reflexionar acerca de las mejoras que se deben introducir, profundizando en su adecuado diseño.

El análisis y la valoración crítica de propuestas están considerados como actividades positivas y recomendadas en la formación de maestros. Con actividades de este tipo los estudiantes son capaces de valorar las propuestas focalizando la atención en aspectos relacionados con la indagación y la naturaleza del conocimiento científico, como las guías de observación, el planteamiento de preguntas o la identificación de pruebas (Abell et al., 1998; Davis, 2006; Duncan et al., 2010).

Una herramienta útil para estudiar situaciones reales de aula con maestros en formación son las grabaciones de vídeo. El análisis de un contexto de aula implica el estudio de una gran variedad de aspectos que, en conjunto, expresarían la complejidad del proceso, ya que en una grabación de vídeo quedan recogidas todas las interacciones que permiten dicho análisis (Aragües, Gil Quílez y De la Gándara, 2014). Las 
investigaciones sobre ese tema ponen de manifiesto que se puede seguir la evolución del análisis de los futuros maestros, que pasaría de una simple evaluación de los conocimientos de los alumnos a un análisis de los razonamientos puestos en práctica por los alumnos entre ellos y con el profesor (Richoux, Tiberghien y Saint Georges, 2012). Esta reflexión facilita a los maestros en formación la mejora en el diseño de propuestas didácticas.

\section{Diseño de la investigación}

\section{Participantes y contexto}

Se ha trabajado con 60 estudiantes de $3^{\text {er }}$ curso del grado en Magisterio de Educación Primaria, que cursan sus estudios en la Facultad de Educación de la Universidad de Zaragoza, en el marco de las actividades de la asignatura Didáctica del medio biológico y geológico. El curso anterior ya habían cursado la asignatura Didáctica del medio físico y químico.

Se grabaron dos vídeos correspondientes a la actuación de dos estudiantes del mismo grado, durante el periodo de Prácticas Escolares III (4ํㅜ curso), que desarrollaron en un colegio concertado de la ciudad, con alumnos de 2 o curso de Educación Primaria. El vídeo I corresponde a una estudiante que trabajó una sesión sobre la evaporación, ciclo del agua y densidad, y el vídeo II corresponde a otra estudiante que abordó una lección sobre el movimiento de rotación terrestre, el día y la noche. Ambos vídeos tratan de temas diferentes, pero las dos sesiones grabadas incluían breves exposiciones de las maestras en prácticas acerca de los contenidos abordados, la manipulación de materiales y la realización de experiencias que podrían considerarse de un bajo nivel de indagación, es decir, del tipo de experiencias confirmatorias planteadas por Windschitl (2003). A partir de su visionado, el objetivo era que los futuros maestros aplicasen sus conocimientos profesionales para analizar unas clases en las que hay una gran interacción entre profesorado y alumnado.

El vídeo I se pasó a los estudiantes al comienzo de la asignatura, y el vídeo II, al finalizar la misma. Después del análisis de los vídeos, los resultados se discutieron en clase utilizando los apartados de la Tabla 1. A lo largo del curso, los estudiantes trabajaron la indagación con actividades prácticas y debían elaborar una propuesta didáctica para aplicarla en un aula de primaria.

Tabla 1. Aspectos considerados por los maestros en formación al analizar clases de ciencias (adaptado de Duncan et al., 2010)

\begin{tabular}{|c|c|}
\hline \multicolumn{2}{|r|}{ CATEGORÍAS } \\
\hline \multicolumn{2}{|r|}{ Objetivos } \\
\hline \multicolumn{2}{|r|}{ Motivación } \\
\hline \multicolumn{2}{|r|}{ Conocimientos previos } \\
\hline \multicolumn{2}{|r|}{ Contextualización } \\
\hline \multicolumn{2}{|r|}{ Constructivismo } \\
\hline \multicolumn{2}{|r|}{ Conexión con otras ideas } \\
\hline \multicolumn{2}{|r|}{ Evaluación } \\
\hline \multirow{3}{*}{ Indagación } & Estructurada \\
\hline & Guiada \\
\hline & Abierta \\
\hline \multirow{3}{*}{ Práctica científica } & Simples observaciones, toma de datos \\
\hline & Análisis e interpretación de datos, evidencias \\
\hline & Experimentos con modificación de variables \\
\hline
\end{tabular}




\section{Metodología de análisis}

Para recoger la información, relativa a cada uno de los vídeos, se proporcionó a los estudiantes un cuestionario basado en Duncan et al. (2010) que se presenta en el Anexo 1. Para el análisis de las respuestas a las preguntas 2 a 4 se establecieron categorías relacionadas con:

- Aspectos relacionados con la estructura general de la sesión: objetivos, carácter motivador de la actividad, que se utilicen los conocimientos previos de los alumnos, abordar la actividad de manera contextualizada, relación con otros contenidos o con la vida cotidiana y todo ello con una visión constructivista del aprendizaje que por supuesto debe ser evaluado (Tabla 1).

- Aspectos relacionados con la indagación y la naturaleza del conocimiento científico: observación, planteamiento de preguntas, uso de pruebas, diseño de experimentos, toma de datos, etc. (Tabla 1 ).

Con respecto a la primera pregunta se agruparon las repuestas según hiciesen referencia a contenidos teóricos o si incluían procedimientos. En la última pregunta, que hacía referencia a propuestas de mejora, se analizó si indicaban mejoras en la metodología o simplemente aportaban sugerencias para mantener la disciplina en la clase.

\section{Contenido de los vídeos}

Los dos vídeos habían sido previamente transcritos y analizados por las autoras de una manera general. Este análisis previo permitió seleccionar los fragmentos relacionados con la enseñanza de las ciencias, suprimiendo gran parte de los periodos dedicados a la organización del aula. En el vídeo I, la maestra en prácticas realizó con el alumnado tres experiencias relacionadas con evaporación y densidad y muy brevemente mencionó el ciclo del agua, a partir de un esquema mostrado en una presentación de ordenador (Tabla 2).

Tabla 2. Temporalización de las actividades del vídeo I, evaporación, ciclo del agua y densidad

\begin{tabular}{|c|c|c|c|c|}
\hline $\begin{array}{l}\text { Tiempo } \\
\text { (min.) }\end{array}$ & Modalidad & Etapas & Estudiante en prácticas & Alumnos/as \\
\hline $\begin{array}{l}00: 00 / \\
01: 36\end{array}$ & $\begin{array}{l}\text { Conjunto de } \\
\text { la clase }\end{array}$ & Introducción & $\begin{array}{l}\text { Pregunta qué es la ciencia y } \\
\text { confirma las respuestas que } \\
\text { considera correctas }\end{array}$ & $\begin{array}{l}\text { Responden a las } \\
\text { preguntas }\end{array}$ \\
\hline $\begin{array}{l}01: 36 / \\
06: 26\end{array}$ & $\begin{array}{l}\text { Conjunto de } \\
\text { la clase }\end{array}$ & $\begin{array}{l}\text { Observación del } \\
\text { resultado }\end{array}$ & $\begin{array}{l}\text { Muestra el plato con sal, } \\
\text { pregunta y cuestiona } \\
\text { respuestas }\end{array}$ & $\begin{array}{l}\text { Pasan en fila para } \\
\text { observar y decir } \\
\text { qué es }\end{array}$ \\
\hline $\begin{array}{l}06: 26 / \\
07: 01\end{array}$ & $\begin{array}{l}\text { Conjunto de } \\
\text { la clase }\end{array}$ & $\begin{array}{l}\text { Organización de } \\
\text { la clase }\end{array}$ & $\begin{array}{l}\text { Organiza la asamblea } \\
\text { sentados en el suelo }\end{array}$ & \\
\hline $\begin{array}{l}07: 01 / \\
08: 30\end{array}$ & $\begin{array}{l}\text { Conjunto de } \\
\text { la clase }\end{array}$ & Exposición & $\begin{array}{l}\text { Muestra el ciclo del agua } \\
\text { con un esquema de } \\
\text { ordenador. } \\
\text { Pregunta }\end{array}$ & $\begin{array}{l}\text { Observan el } \\
\text { esquema del ciclo } \\
\text { del agua }\end{array}$ \\
\hline $\begin{array}{l}08: 30 / \\
11: 38 \\
\end{array}$ & $\begin{array}{l}\text { Trabajo en } \\
\text { equipo }\end{array}$ & $\begin{array}{l}\text { Organización del } \\
\text { aula }\end{array}$ & $\begin{array}{l}\text { Organiza grupos de } 4 \\
\text { alumnos }\end{array}$ & \\
\hline $\begin{array}{l}11: 38 / \\
24: 56\end{array}$ & $\begin{array}{l}\text { Trabajo en } \\
\text { equipo }\end{array}$ & $\begin{array}{l}\text { Realización de la } \\
\text { experiencia }\end{array}$ & $\begin{array}{l}\text { Distribuye vasos, agua con } \\
\text { sal y sin sal y un huevo en } \\
\text { cada vaso. }\end{array}$ & $\begin{array}{l}\text { Observan, } \\
\text { comentan, } \\
\text { escriben, dibujan. }\end{array}$ \\
\hline
\end{tabular}


Tabla 2. Continuación

\begin{tabular}{|c|c|c|c|c|}
\hline $\begin{array}{l}\text { Tiempo } \\
\text { (min.) }\end{array}$ & Modalidad & Etapas & Estudiante en prácticas & Alumnos/as \\
\hline $\begin{array}{l}24: 56 / \\
26: 43\end{array}$ & $\begin{array}{l}\text { Conjunto de } \\
\text { la clase }\end{array}$ & $\begin{array}{l}\text { Organización del } \\
\text { aula }\end{array}$ & $\begin{array}{l}\text { Reorganiza la asamblea al } \\
\text { fondo del aula }\end{array}$ & $\begin{array}{l}\text { Se desplazan al } \\
\text { fondo del aula }\end{array}$ \\
\hline $\begin{array}{l}26: 43 / \\
28: 50\end{array}$ & $\begin{array}{l}\text { Conjunto de } \\
\text { la clase } \\
\text { Debate }\end{array}$ & $\begin{array}{l}\text { Predicción sobre } \\
\text { nueva experiencia }\end{array}$ & $\begin{array}{l}\text { Propone nueva experiencia: } \\
\text { un vaso con agua y un } \\
\text { huevo, ¿qué pasará al } \\
\text { agregar sal? }\end{array}$ & $\begin{array}{l}\text { Hacen } \\
\text { comentarios } \\
\text { diversos }\end{array}$ \\
\hline $\begin{array}{l}28: 50 / \\
29: 56 \\
\end{array}$ & $\begin{array}{l}\text { Trabajo en } \\
\text { equipo }\end{array}$ & $\begin{array}{l}\text { Reorganización } \\
\text { del aula }\end{array}$ & $\begin{array}{l}\text { Reorganiza los alumnos y } \\
\text { las mesas }\end{array}$ & $\begin{array}{l}\text { Vuelven a las } \\
\text { mesas }\end{array}$ \\
\hline $\begin{array}{l}29: 56 / \\
36: 25\end{array}$ & $\begin{array}{l}\text { Trabajo en } \\
\text { equipo }\end{array}$ & Nueva experiencia & $\begin{array}{l}\text { Reparte agua y sal en los } \\
\text { vasos y confirma que flota } \\
\text { el huevo }\end{array}$ & $\begin{array}{l}\text { Ponen sal y } \\
\text { remueven el agua }\end{array}$ \\
\hline $\begin{array}{l}36: 25 / \\
38: 10\end{array}$ & $\begin{array}{l}\text { Conjunto de } \\
\text { la clase }\end{array}$ & $\begin{array}{l}\text { Organización del } \\
\text { aula }\end{array}$ & $\begin{array}{l}\text { Reorganiza la asamblea al } \\
\text { fondo del aula }\end{array}$ & \\
\hline $\begin{array}{l}38: 10 / \\
38: 45\end{array}$ & $\begin{array}{l}\text { Conjunto de } \\
\text { la clase }\end{array}$ & Comentario & $\begin{array}{l}\text { Pregunta por lo observado } \\
\text { y confirma }\end{array}$ & $\begin{array}{l}\text { Responden que el } \\
\text { huevo se ha ido } \\
\text { hacia arriba }\end{array}$ \\
\hline $\begin{array}{l}38: 45 / \\
39: 38\end{array}$ & $\begin{array}{l}\text { Conjunto de } \\
\text { la clase }\end{array}$ & Explicación & Explica el experimento & $\begin{array}{l}\text { Escuchan y } \\
\text { repiten } \\
\text { explicación }\end{array}$ \\
\hline $\begin{array}{l}39: 38 / \\
40: 06\end{array}$ & $\begin{array}{l}\text { Conjunto de } \\
\text { la clase }\end{array}$ & Aplicación & $\begin{array}{l}\text { ¿Flotará mejor en el río que } \\
\text { en el mar? }\end{array}$ & $\begin{array}{l}\text { Responden } \\
\text { preguntas }\end{array}$ \\
\hline $\begin{array}{l}40: 06 / \\
40: 30\end{array}$ & $\begin{array}{l}\text { Conjunto de } \\
\text { la clase }\end{array}$ & $\begin{array}{l}\text { Evaluación de la } \\
\text { clase }\end{array}$ & $\begin{array}{l}\text { Pregunta si les gustó la } \\
\text { clase y se queja de su } \\
\text { comportamiento }\end{array}$ & $\begin{array}{l}\text { Responden que } \\
\text { les gustó la clase }\end{array}$ \\
\hline
\end{tabular}

La clase registrada en el vídeo Il se refirió al movimiento de rotación de la tierra y la consecuente sucesión de días y noches en los diversos lugares del planeta. La maestra en prácticas realizó, al inicio de la clase, una explicación dialogada sobre el movimiento de rotación en base a una lámina, y posteriormente tres experiencias diferentes con el alumnado (Tabla 3).

Tabla 3. Temporalización de las actividades del vídeo II, el día y la noche y la rotación de la tierra

\begin{tabular}{|l|l|l|l|l|}
\hline $\begin{array}{l}\text { Tiempo } \\
\text { (min.) }\end{array}$ & Modalidad & Etapas & Estudiante en Prácticas & Alumnos/as \\
\hline $\begin{array}{l}\text { 00:00 / } \\
\text { 01:30 }\end{array}$ & $\begin{array}{l}\text { Conjunto de } \\
\text { la clase }\end{array}$ & Evaluación & $\begin{array}{l}\text { Pregunta qué es la ciencia y } \\
\text { confirma las respuestas que } \\
\text { considera correctas }\end{array}$ & $\begin{array}{l}\text { Responden a } \\
\text { las preguntas }\end{array}$ \\
\hline $\begin{array}{l}\text { 01:30 / } \\
\text { 03:06 }\end{array}$ & $\begin{array}{l}\text { Conjunto de } \\
\text { la clase }\end{array}$ & Explicación & $\begin{array}{l}\text { Pregunta qué es la tierra, } \\
\text { relación con el sol y rotación. }\end{array}$ & $\begin{array}{l}\text { Responden a } \\
\text { las preguntas }\end{array}$ \\
\hline $\begin{array}{l}\text { 03:06 / } \\
\text { 04:30 }\end{array}$ & $\begin{array}{l}\text { Conjunto de } \\
\text { la clase }\end{array}$ & Explicación & $\begin{array}{l}\text { Comenta la duración y } \\
\text { velocidad de la rotación }\end{array}$ & $\begin{array}{l}\text { Responden a } \\
\text { las preguntas }\end{array}$ \\
\hline $\begin{array}{l}\text { 04:30 / } \\
\text { 10:49 }\end{array}$ & $\begin{array}{l}\text { Conjunto de } \\
\text { la clase }\end{array}$ & $\begin{array}{l}\text { Organización de la } \\
\text { clase }\end{array}$ & $\begin{array}{l}\text { Organiza la asamblea en } \\
\text { parejas y entrega una } \\
\text { linterna a cada una }\end{array}$ & $\begin{array}{l}\text { Se desplazan al } \\
\text { fondo del aula }\end{array}$ \\
\hline $\begin{array}{l}\text { 10:49 / } \\
\text { 12:45 }\end{array}$ & $\begin{array}{l}\text { Trabajo en } \\
\text { equipo }\end{array}$ & $\begin{array}{l}\text { Consignas para } \\
\text { realización de } \\
\text { experiencia }\end{array}$ & $\begin{array}{l}\text { Un alumno será el sol, } \\
\text { iluminará con la linterna al } \\
\text { compañero quien girará } \\
\text { sobre sí mismo }\end{array}$ & \\
\hline
\end{tabular}


Tabla 3. Continuación

\begin{tabular}{|c|c|c|c|c|}
\hline $\begin{array}{l}\text { Tiempo } \\
\text { (min.) }\end{array}$ & Modalidad & Etapas & Estudiante en Prácticas & Alumnos/as \\
\hline $\begin{array}{l}12: 45 / \\
14: 29\end{array}$ & $\begin{array}{l}\text { Trabajo en } \\
\text { equipo }\end{array}$ & $\begin{array}{l}\text { Realización de la } \\
\text { experiencia }\end{array}$ & $\begin{array}{l}\text { Destaca la necesidad de } \\
\text { observar para ser científicos }\end{array}$ & $\begin{array}{l}\text { Realizan la } \\
\text { simulación }\end{array}$ \\
\hline $\begin{array}{l}14: 29 / \\
15: 50\end{array}$ & $\begin{array}{l}\text { Trabajo en } \\
\text { equipo }\end{array}$ & $\begin{array}{l}\text { Realización de la } \\
\text { experiencia }\end{array}$ & Indica que cambien de rol & $\begin{array}{l}\text { Quien era sol, pasa } \\
\text { a ser tierra y } \\
\text { viceversa }\end{array}$ \\
\hline $\begin{array}{l}15: 50 / \\
16: 47\end{array}$ & $\begin{array}{l}\text { Conjunto de } \\
\text { la clase }\end{array}$ & $\begin{array}{l}\text { Organización del } \\
\text { aula }\end{array}$ & Reorganiza a los alumnos & $\begin{array}{l}\text { Se sientan en el } \\
\text { suelo }\end{array}$ \\
\hline $\begin{array}{l}16: 47 / \\
17: 15\end{array}$ & $\begin{array}{l}\text { Conjunto de } \\
\text { la clase }\end{array}$ & $\begin{array}{l}\text { Comentarios } \\
\text { sobre la } \\
\text { observación }\end{array}$ & $\begin{array}{l}\text { Pregunta por lo simulado, } \\
\text { confirma las respuestas que } \\
\text { considera correctas }\end{array}$ & $\begin{array}{l}\text { Responden a las } \\
\text { preguntas }\end{array}$ \\
\hline $\begin{array}{l}17: 15 / \\
17: 51\end{array}$ & $\begin{array}{l}\text { Conjunto de } \\
\text { la clase }\end{array}$ & $\begin{array}{l}\text { Explicación } \\
\text { dialogada }\end{array}$ & $\begin{array}{l}\text { Pregunta sobre zonas } \\
\text { iluminadas y oscuras para } \\
\text { identificarlas como día y } \\
\text { noche }\end{array}$ & $\begin{array}{l}\text { Responden a las } \\
\text { preguntas }\end{array}$ \\
\hline $\begin{array}{l}17: 51 / \\
18: 18\end{array}$ & $\begin{array}{l}\text { Conjunto de } \\
\text { la clase }\end{array}$ & $\begin{array}{l}\text { Generalización } \\
\text { dialogada }\end{array}$ & $\begin{array}{l}\text { Generaliza que gracias a la } \\
\text { luz del sol tenemos día y } \\
\text { noche en los diferentes } \\
\text { países del mundo }\end{array}$ & $\begin{array}{l}\text { Responden a las } \\
\text { preguntas }\end{array}$ \\
\hline $\begin{array}{l}18: 18 / \\
29: 08\end{array}$ & $\begin{array}{l}\text { Conjunto de } \\
\text { la clase }\end{array}$ & $\begin{array}{l}\text { Predicción } \\
\text { dialogada }\end{array}$ & $\begin{array}{l}\text { Pregunta en qué países } \\
\text { están de noche si en España } \\
\text { es de día, usan el globo } \\
\text { terráqueo }\end{array}$ & $\begin{array}{l}\text { Responden a } \\
\text { preguntas de la } \\
\text { estudiante en } \\
\text { prácticas }\end{array}$ \\
\hline $\begin{array}{l}29: 08 / \\
29: 51 \\
\end{array}$ & $\begin{array}{l}\text { Conjunto de } \\
\text { la clase }\end{array}$ & Síntesis & $\begin{array}{l}\text { Sintetiza oralmente los } \\
\text { contenidos abordados }\end{array}$ & \\
\hline $\begin{array}{l}29: 51 / \\
30: 24\end{array}$ & $\begin{array}{l}\text { Conjunto de } \\
\text { la clase }\end{array}$ & $\begin{array}{l}\text { Consigna de } \\
\text { registro }\end{array}$ & $\begin{array}{l}\text { Indica que registren en sus } \\
\text { libretas qué es el día y qué } \\
\text { es la noche y que dibujen }\end{array}$ & \\
\hline
\end{tabular}

\section{Resultados}

Los estudiantes contestaron al cuestionario y posteriormente se discutieron en clase las valoraciones realizadas y los aspectos en los que se habían fijado, reflexionando sobre las dificultades detectadas. Las respuestas fueron amplias y no se centraron solo en cuestiones generales del tipo: "La interacción profesora-alumnos es constante", "El lenguaje está adaptado al nivel de los niños", "Les da un feed-back positivo", "Apoyar todo lo que va explicando con medios audiovisuales como fotos o vídeos", "Ha sido una sesión muy planificada previamente", "Estaba bien estructurada y ajustada al tiempo", "Creo que es muy beneficioso el trabajo en grupo", "Creo que son alumnos muy pequeños", sino que muchas respuestas hacían referencia a aspectos concretos de enseñanza por indagación: "No les permite experimentar y es la base del aprendizaje indagatorio", "Sí que observan y experimentan, pero no investigan porque es la profesora la que va realizando los pasos y la que da las explicaciones".

Como se ha señalado, para analizar las respuestas de los estudiantes nos basamos en la red de análisis presentada por Duncan et al. (2010). Estos autores establecen unas categorías centradas en la estructura de la sesión, si se hace referencia a los objetivos, a los conocimientos previos, a la evaluación, etc., y otras centradas en la indagación, distinguiendo si hacían referencia a distintos niveles de autonomía del alumnado en la 
indagación, a las prácticas científicas y la naturaleza de la ciencia. De esta manera, para la categoría de objetivos o de evaluación analizábamos si los estudiantes hacían referencia a ellos: "Yo creo que el objetivo fundamental de la profesora de prácticas seguramente fuese que los alumnos investigasen", "No ha realizado una evaluación de la actividad, sin ella no sabrá si se han cumplido los objetivos programados".

Los resultados del análisis de las respuestas al cuestionario tras el visionado de ambos vídeos están reflejados en la Tabla 4. En algunas categorías no hay apenas variaciones en las respuestas de los estudiantes en ambos cuestionarios, como en objetivos, motivación, constructivismo; en otras hay un incremento que no resulta relevante, excepto en las referencias a la de indagación que se trabajó a lo largo del curso.

Tabla 4. Número de respuestas correspondientes a cada categoría

\begin{tabular}{|c|c|c|c|}
\hline \multicolumn{2}{|r|}{ CATEGORÍAS } & INICIAL & FINAL \\
\hline \multicolumn{2}{|r|}{ Objetivos } & 2 & 4 \\
\hline \multicolumn{2}{|r|}{ Motivación } & 16 & 13 \\
\hline \multicolumn{2}{|r|}{ Conocimientos previos } & 6 & 22 \\
\hline \multicolumn{2}{|r|}{ Contextualización } & 7 & 2 \\
\hline \multicolumn{2}{|r|}{ Constructivismo } & 32 & 35 \\
\hline \multicolumn{2}{|r|}{ Conexión con otras ideas } & 0 & 1 \\
\hline \multicolumn{2}{|r|}{ Evaluación } & 4 & 11 \\
\hline \multirow{3}{*}{ Indagación } & Estructurada & 13 & 43 \\
\hline & Guiada & 2 & 11 \\
\hline & Abierta & 0 & 8 \\
\hline \multirow{3}{*}{ Práctica científica } & Simples observaciones, toma de datos & 15 & 24 \\
\hline & Análisis e interpretación de datos, evidencias & 3 & 1 \\
\hline & Experimentos con modificación de variables & 2 & 9 \\
\hline
\end{tabular}

En las discusiones de los resultados de los dos vídeos los estudiantes coincidían en valorar positivamente la actividad, ya que les enfrentaba con situaciones de aula reales con las que se sentían identificados por tratarse de estudiantes en prácticas. Percibían, además, los numerosos aspectos que inciden en las actividades de aula y de los que hay que ser conscientes para que el proceso de enseñanza aprendizaje llegue a buen puerto: "Este vídeo es superfuerte, ahora me doy cuenta de todas las cosas que tengo que controlar para que aprendan".

\section{Constructivismo}

Como en los cursos previos los futuros maestros han recibido una formación psicopedagógica, ya habían trabajado los fundamentos constructivistas. Queríamos averiguar de qué manera integraban los presupuestos constructivistas con el aprendizaje de un tema concreto de ciencias.

En sus respuestas, más de la mitad de los estudiantes hacen referencia al constructivismo en ambos cuestionarios (Tabla 4), aunque se refieren a él de una manera un tanto ingenua, utilizando frases hechas, sin desarrollar, señalan principalmente que el alumno es protagonista de su propio aprendizaje:

"El papel de los alumnos es activo facilitando la construcción del propio aprendizaje, siendo éste significativo".

"Permite que sean los niños los que detecten, describan y analicen lo que se les presenta para que sean ellos mismos quienes, a partir de lo que saben, construyan sus primeras explicaciones". 
En ninguno de los dos vídeos las maestras en prácticas evalúan los aprendizajes, sólo buscan respuestas correctas y les basta con que un único alumno la proporcione. No hay una interacción sistemática con los distintos alumnos que vaya guiando su razonamiento para la elaboración de una explicación de los fenómenos.

Por otro lado, en el vídeo se ve a los niños de Primaria muy activos, pero solo el hecho de estar manipulando no implica necesariamente que están aprendiendo, simplemente realizan una tarea que no siempre deja poso en cuanto al conocimiento científico, ni teórico ni de procedimientos o actitudes.

En cuanto a la contextualización, relacionar los temas con la vida cotidiana de los alumnos, hacerlos atractivos, motivar, partir de los conocimientos previos, aspectos que favorecen que el alumno se implique en el aprendizaje y vaya construyendo conocimiento, no son aspectos que se destaquen en la actuación de las maestras en prácticas. Sin embargo, al analizarlos los futuros maestros señalan que las actividades desarrolladas en los vídeos resultan atractivas ya que ven a los niños y niñas manipulando linternas, vasos, huevos, etc., en un ambiente muy lúdico. Pero las actividades de los vídeos apenas se quedan en la novedad de ese ambiente lúdico, sin que haya evidencias de que se haya producido un aprendizaje significativo.

El constructivismo implica una actividad social, con interacción tanto entre los alumnos como entre estos y el maestro; con un espacio para la discusión, reflexión e imaginación. En definitiva, facilitar la construcción de nuevo conocimiento por parte del alumnado, guiados por el maestro. Todos estos aspectos ligados al constructivismo están ausentes en las actividades de las sesiones y apenas son comentados por los futuros maestros al analizar los vídeos.

\section{Práctica científica}

Lo que caracteriza a las prácticas científicas es la actividad que se realiza. Los alumnos deben experimentar, argumentar, modelizar, comunicar en lugar de simplemente manipular (Jiménez-Aleixandre y Crujeiras, 2016). El objetivo de las maestras en los videos era planificar y realizar una pequeña investigación, es decir, llevar a cabo una práctica científica. Esto implica diseñar la experimentación y la toma de datos, identificando pruebas, interpretándolas y comunicando los resultados. Pero finalmente realizan una serie de actividades manipulativas en las que los alumnos de primaria no tienen oportunidades para plantear preguntas, hipótesis y construir explicaciones. Sin embargo las respuestas de los futuros maestros no siempre hacen referencia a esa falta de oportunidades; al contrario:

"De una forma guiada y a través de los experimentos sí que investigan ya que tienen oportunidad de observar, hacer hipótesis y descubrir si son verdaderas".

"Sí, porque se formula una hipótesis por los propios alumnos sobre lo que se cree que va a pasar. Se realiza un experimento sobre el tema para contrastar la hipótesis. Y se comprueba si la hipótesis es cierta y no".

Parece que los futuros maestros encuestados no tienen claro qué es una hipótesis, y la confunden con las respuestas espontáneas de los alumnos de primaria a las cuestiones cerradas planteadas por las maestras. Cuando un alumno da la respuesta que la maestra considera correcta la da por buena y sigue la clase dando por finalizada la discusión. No continúa la interacción con el resto de la clase para asegurarse que efectivamente todos los alumnos están entendiendo el proceso.

Los futuros maestros consideran que una actividad experimental ya es hacer práctica 
científica. Al analizar las respuestas de los alumnos se ve que no hay diferencias significativas entre los análisis del video inicial y final (Tabla 4). Sin embargo, en el primero la maestra en prácticas lleva a cabo una secuencia de actividades que podría ser considerada como práctica científica, aunque la mayor parte del protagonismo de la realización recae sobre ella más que sobre los alumnos.

\section{Indagación}

Como era de esperar, los análisis mejoran en el cuestionario final (Tabla 4), ya que durante el curso se trabajó la metodología de indagación. Algunas de las referencias a la indagación son sencillas:

"Yo diría, que en lugar de investigar, más bien experimentan y descubren..."

Pero en ocasiones, las respuestas del cuestionario final son más elaboradas haciendo referencia al gran protagonismo asumido por la maestra en prácticas en los distintos aspectos de la clase (problema, preguntas, experiencia, variables...):

"No sería una actividad plenamente de indagación, sino que estaría en uno de los niveles más básicos, cuando la docente marca más que guía y el alumno se limita a responder más que a participar como científico. Esto me parece así porque: 1) Los alumnos no plantean preguntas, sino que se las hacen; 2) Los alumnos no proponen el diseño experimental; 3) Los alumnos no piensan sobre posibles variables y cómo influirán; 4) Se parte de un fenómeno (día y noche), no de un problema o pregunta que "obligue" a indagar o investigar o experimentar para responder".

Por otra parte, aunque no lo dicen explícitamente, los futuros maestros hacen referencia a la indagación estructurada considerando que no es propiamente investigación:

"Yo creo que no se trata de una investigación, sino de una experimentación muy guiada".

Hay que señalar que se había discutido en clase que la indagación debe pasar por distintos niveles, desde muy estructurada hasta llegar a niveles en los que los alumnos estén capacitados para realizar una indagación abierta (Windschitl, 2003).

\section{Temas de ciencia abordados}

El análisis de los temas que, a criterio de los docentes en formación, fueron tratados en las clases registradas, brinda información acerca de cómo comprenden lo que es un contenido enseñado. Permite asimismo evidenciar la posibilidad de identificación de contenidos procedimentales, como proceso que se debe enseñar al alumnado.

Nunca se hace referencia de manera directa a la naturaleza de la ciencia, pero hemos considerado que queda implícita cuando señalan que no sólo se han trabajado contenidos conceptuales sino la observación o el método científico; o se hace referencia a qué es la ciencia o qué son los científicos.

En el análisis del primer vídeo, un $85 \%$ hace referencia a que se han trabajado contenidos conceptuales (ciclo del agua, densidad, flotabilidad, evaporación, etc.) y solo el $12 \%$ indican aspectos relacionados con la ciencia y el trabajo de los científicos (observación, método científico, etc.). En el segundo vídeo los resultados son semejantes; el $13 \%$ mencionan la observación, experimentación, etc., como temas de ciencia tratados en el vídeo. Consideran mayoritariamente que aprender ciencia es aprender contenidos conceptuales y no aspectos relacionados con la práctica científica. 
Siendo que la ciencia y cómo trabajan los científicos fueron explícitamente mencionados en los vídeos por las maestras al comienzo de la clase, llama la atención el bajo porcentaje de estudiantes que los consideran contenidos de ciencia.

\section{Propuestas de mejora}

Mayoritariamente las propuestas de mejora, en los dos vídeos, se centran en aspectos organizativos del aula (Tabla 5): tipo de material, ambiente ruidoso y falta de disciplina.

"Yo les daría una bola del mundo más grande; modificaría el tono de voz, tanto de la maestra como de los alumnos, había mucho barullo".

La organización de la clase y el ambiente de aula es un aspecto importante de la metodología de indagación que en ella se aplica o que se pretende aplicar. El ambiente ruidoso e incluso algo caótico es inherente a la puesta en marcha de metodologías nuevas como la indagación y es algo por lo que hay que pasar, hasta que el alumnado de primaria haya adquirido las rutinas de trabajo adecuadas.

Tabla 5. Propuestas de mejora señaladas por los maestros en formación al analizar clases

\begin{tabular}{|l|l|c|c|}
\hline \multicolumn{2}{|c|}{ CATEGORÍAS } & INICIAL & FINAL \\
\hline \multirow{2}{*}{ Propuestas de mejora } & en la metodología de indagación & 14 & 27 \\
\cline { 2 - 4 } & en la organización de la clase & 49 & 38 \\
\hline
\end{tabular}

Sin embargo, los estudiantes no se fijan en aspectos especialmente llamativos como que las maestras invierten mucho tiempo en mover a los alumnos de un lugar a otro de la clase, en el reparto del material, y que este cambio rompe el hilo conductor de la clase.

\section{Conclusiones e implicaciones didácticas}

Aunque los vídeos utilizados no constituyen una ejemplificación ideal de lo que podría ser una sesión de clase de enseñanza por indagación, los alumnos perciben las actuaciones de aula analizadas como algo próximo y factible, por lo que encuentran interesantes este tipo de actividades y se implican en la observación y análisis de las sesiones grabadas. Consideramos por tanto que constituyen una buena herramienta de discusión y reflexión, ya que una adecuada comprensión de lo que ocurre en el aula permite plantear aportaciones para la mejora curricular, en lo que concierne a la formación de maestros.

Los futuros maestros no están acostumbrados a hacer prácticas científicas y eso repercute en los planteamientos de la enseñanza por indagación y en el análisis de las mismas. Es por ello que una sugerencia de mejora de nuestro propio trabajo es la necesidad de trabajar las unidades de los vídeos posteriormente a su análisis. Así se les podría pedir a los estudiantes la elaboración detallada de propuestas sobre la temática de las sesiones, con el objetivo de solventar los problemas detectados.

En los vídeos llama la atención que las actividades están guiadas por preguntas planteadas fundamentalmente por las maestras en prácticas, dejando poco protagonismos a los alumnos de primaria. Se trata de preguntas mayoritariamente finalistas, es decir, que buscan la respuesta correcta, y una vez que se obtiene se pasa a otra cuestión. Por tanto un aspecto a trabajar con los futuros maestros es diseñar baterías de preguntas adecuadas para cada momento del proceso y que sirvan para guiar al alumno en su aprendizaje.

Un aspecto importante en el aprendizaje es crear un ambiente de aula agradable y motivador. Circunstancia que está relacionada con la propuesta de una organización 
más eficiente del espacio y de los materiales desde el principio y con el seguimiento de unas pautas generales basadas en la Tabla 1. Así, es necesario insistir en que los alumnos de primaria deberían conocer desde el principio cuál es la pregunta, el problema de partida y por tanto el objetivo de la actividad.

Para finalizar, queremos señalar que enfatizamos con los estudiantes de magisterio el valor del trabajo realizado por estas maestras noveles en prácticas. Habían planteado para sus propuestas didácticas un nivel bajo de indagación, es decir, experiencias de confirmación, dado que iban a trabajar con alumnos que no estaban acostumbrados a realizar experiencias en el aula. Su actuación ha sido valiente e innovadora puesto que se trataba de abrir nuevos caminos con esos niños y niñas. Como siempre que se empieza algo nuevo se encuentran obstáculos, aspectos a mejorar y cambiar, y hay que ser conscientes de que si somos docentes siempre tenemos que estar aprendiendo.

\section{Agradecimientos}

Al proyecto EDU2016-76743-P financiado por el Ministerio de Economía y Competitividad, y al Grupo de Investigación BEAGLE, perteneciente al Instituto de Investigación de Ciencias Ambientales (IUCA). A las maestras en prácticas y estudiantes participantes en el estudio.

\section{Referencias bibliográficas}

Abell, S. K., Anderson, M. A. y Bryan, L. A. (1998). Investigating preservice elementary science teacher reflective thinking using integrated media case-based instruction in elementary science teacher preparation. Science Education, 82(4), 491-509.

Abril, A. M., Ariza, M. R. , Quesada, A. y García, F .J. (2014) Creencias del profesorado en ejercicio y en formación sobre el aprendizaje por investigación. Revista Eureka sobre Enseñanza y Divulgación de las Ciencias, 11, 1: 22-33

Acevedo Díaz, J. A. (2008). El estado actual de la naturaleza de la ciencia en la didáctica de las ciencias. Revista Eureka sobre Enseñanza y Divulgación de las Ciencias, 5(2), 133-169.

Aragües, A., Gil Quílez, M. J. y De la Gándara, M. (2014). Análisis del papel de los maestros en el desarrollo de actividades de indagación en el practicum de primaria. Didáctica de las ciencias experimentales y sociales, 28, 135-151.

Cañal P. (2007). La investigación escolar, hoy. Alambique Didáctica de las Ciencias Experimentales, 24, 46-47.

Cortés Gracia, A. L. y De La Gándara Gómez, M. (2006). La construcción de problemas en el laboratorio durante la formación de profesorado: una experiencia didáctica. Enseñanza de las Ciencias, 25(3), 435-450.

Cortés Gracia, A. L., De la Gándara Gómez, M., Calvo Hernández, J. M., Martínez Peña, M. B., Ibarra Murillo, J., Arlegui de Pablos, J. y Gil Quílez, M. J. (2012). Expectativas, necesidades y oportunidades de los maestros en formación ante la enseñanza de las ciencias en la educación primaria. Enseñanza de las Ciencias, 30(3), 155-176.

Crujeiras, B. y Puig, B. (2014). Trabajar la naturaleza de la ciencia en la formación inicial del profesorado planificando una investigación. Educació Química, 17, 55-61.

Crujeiras, B. y Jiménez-Aleixandre, M. P. (2015). Desafíos planteados por las actividades 
abiertas de indagación en el laboratorio: articulación de conocimientos teóricos y prácticos en las prácticas científicas. Enseñanza de las Ciencias, 33(1), 63-84.

Cuevas, P., Lee, O., Hart, J. y Deakort, R. (2005). Improving science inquiry with elementary students of diverse backgrounds. Journal of Research in Science Teaching, 42(3), 337-357.

Davis, E. A. (2006). Preservice elementary teachers' critique of instructional materials for science. Science Education, 90, 348-375.

Duncan, R. G., Pilitsis, V. y Piegaro, M. (2010). Development of preservice teachers' ability to critique and adapt inquiry-based instructional materials. Journal of Science Teacher Education, 21(1), 81-102.

Gil Quílez M. J., Martínez Peña, M. B., De la Gándara Gómez, M., Calvo Hernández, J.M. y Cortés Gracia, A. L. (2008). De la universidad a la escuela: no es fácil la indagación científica. Revista Interuniversitaria de Formación del Profesorado, 22(3), 81-100.

Guisasola, J. y Morentín, M. (2007). ¿Comprenden la naturaleza de la ciencia los futuros maestros y maestras de Educación Primaria? Revista Electrónica de Enseñanza de las Ciencias, 6(2), 246-262.

Jiménez-Aleixandre, M. P. y Crujeiras, B. (2016). Epistemic practices and scientific practices in science education. En K. S. Taber y B. Akpan (Eds.), Science Education: An International Course Companion, pp. 69-80. Rotterdam, The Netherlands: Sense Publishers.

Kirschner, P. A., Sweller, J. y Clark, R. E. (2006). Why Minimal Guidance During Instruction Does Not Work: An Analysis of the Failure of Constructivist, Discovery, Problem-Based, Experiential, and Inquiry-Based Teaching. Educational psychologist, 41(2), 75-86.

Lucero, M., Valcke, M. y Schellens, T. (2013). Teachers' beliefs and self-reported use of inquiry in Science Education in Public Primary Schools. International Journal of Science Education, 35 (8), 1407-1423. DOI: https://dx.doi.org/10.1080/ $\underline{09500693.2012 .704430}$

Mcdonald, S. y Butler Songer, N. (2008). Enacting classroom inquiry: Theorizing teachers' conceptions on science teaching. Science Education, 27, 45-60.

MEC, Ministerio de Educación y Ciencia (2007). Orden ECI/2211/2007, de 12 de julio, por la que se establece el currículo y se regula la ordenación de la Educación primaria. BOE núm. 173, 31487- 31566.

NATIONAL RESEARCH COUNCIL (2000). Inquiry and the National Science Education Standards: a guide for teaching and learning. Washington, D.C.: National Academy Press.

Richoux, H., Tiberghien, A. y Saint Georges, M. (2012). Cómo aprenden los profesores a partir de vídeos de alumnos. Enseñanza de las Ciencias, 30(1), 035-048

Rocard, M., Csermely, P., Jorde, D., Lenzen, D., Walwerg-Henriksson, H. y Hemmo, V. (2007). Science Education Now: A Renewed Pedagogy for the Future of Europe. European Commission. Community Research.

Vázquez Alonso, A., Acevedo Díaz, J. A. y Manassero Mas, M. A. (2004). Consensos sobre 
la naturaleza de la ciencia: evidencias e implicaciones para su enseñanza. Revista Iberoamericana de Educación, edición electrónica, http://www.rieoei.org/ deloslectores/ 702Vazquez.PDF. Consultado el 06-03 2017.

Vílchez González, J. M. y Bravo Torija, B. (2015) Percepción del profesorado de ciencias de educación primaria en formación acerca de las etapas y acciones necesarias para realizar una indagación escolar. Enseñanza de las Ciencias, 33(1), 185-202.

Windschitl, M. (2003). Inquiry projects in science teacher education: What can investigative experiences reveal about teacher thinking and eventual classroom practice? Science Education, 87(1), 112-143. 


\section{Anexo 1}

Observa con atención el vídeo. Después de verlo realiza individualmente un análisis del mismo respondiendo a las siguientes cuestiones:

1. Temas de ciencias abordados.

2. ¿Cuáles son las tres cosas que opinas están bien acerca de la lección? ¿Por qué crees que están bien?

3. ¿Cuáles son los tres aspectos que consideras que son problemáticos? (elegir los más problemáticos si hay más de tres). ¿Por qué constituyen un problema?

4. ¿Es esta una lección en la que los alumnos investigan? Explica tu respuesta.

5. ¿Sugerirías algún cambio en relación con la actividad de investigación de los alumnos? Explica en qué mejorarían la clase estos cambios. 